\title{
Determinants of exercise-induced pulmonary arterial hypertension in systemic sclerosis
}

\author{
Damien Voilliot a , Julien Magne a , Raluca Dulgheru ${ }^{a}$, Seisyou Kou ${ }^{\text {a }}$, Christine Henri ${ }^{\text {a }}$, Saloua Laaraibi ${ }^{\text {a }}$, \\ Muriel Sprynger ${ }^{\text {a }}$, Béatrice Andre ${ }^{\mathrm{b}}$, Luc A. Pierard ${ }^{\mathrm{a}, *}$, Patrizio Lancellotti ${ }^{\mathrm{a}, *}$ \\ a University of Liège Hospital, GIGA Cardiovascular Sciences, Department of Cardiology, Heart Valve Clinic, University Hospital Sart Tilman, Liège, Belgium \\ b University of Liège Hospital, Department of Rheumatology, University Hospital Sart Tilman, Liège, Belgium
}

\section{A R T I C L E I N F O}

\section{Article history:}

Received 1 October 2013

Received in revised form 25 January 2014

Accepted 22 February 2014

Available online 28 February 2014

\section{Keywords:}

Scleroderma

Echocardiography

Pulmonary hypertension

Exercise

\begin{abstract}
A B S T R A C T
Background: Exercise-induced pulmonary arterial hypertension (EIPH) in systemic sclerosis (SSc) has already been observed but its determinants remain unclear. The aim of this study was to determine the incidence and the determinants of EIPH in SSc.

Methods and results: We prospectively enrolled 63 patients with SSc (age $54 \pm 3$ years, 76\% female) followed in CHU Sart-Tilman in Liège. All patients underwent graded semi-supine exercise echocardiography. Systolic pulmonary arterial pressure (SPAP) was derived from the peak velocity of the tricuspid regurgitation jet and adding the estimation of right atrial pressure, both at rest and during exercise. Resting pulmonary arterial hypertension ( $\mathrm{PH}$ ) was defined as SPAP $>35 \mathrm{~mm} \mathrm{Hg}$ and EIPH as SPAP $>50 \mathrm{~mm} \mathrm{Hg}$ during exercise. The following formulas were used: mean PAP $(\mathrm{mPAP})=0.61 \times \mathrm{sPAP}+2$, left atrial pressure $(\mathrm{LAP})=1.9+1.24 \times$ left ventricular $(\mathrm{LV}) \mathrm{E} / \mathrm{e}^{\prime}$ and pulmonary vascular resistance $(\mathrm{PVR})=(\mathrm{mPAP}-\mathrm{LAP}) / \mathrm{LV}$ cardiac output $(\mathrm{CO})$ and slope of mPAP-LVCO relationship = changes in mPAP / changes in LVCO. Resting PH was present in 3 patients (7\%) and 21 patients developed EIPH (47\%). Patients with EIPH had higher resting LAP (10.3 \pm 2.2 versus $8.8 \pm 2.3 \mathrm{~mm} \mathrm{Hg;} \mathrm{p}=0.03)$, resting PVR ( $2.6 \pm 0.8$ vs. $1.4 \pm 1.1$ Woods units; $\mathrm{p}=0.004)$, exercise LAP $(13.3 \pm 2.3$ vs. $9 \pm 1.7 \mathrm{~mm} \mathrm{Hg} ; \mathrm{p}<0.0001)$, exercise PVR ( $3.6 \pm 0.7$ vs. $2.1 \pm 0.9$ Woods units; $\mathrm{p}=0.02)$ and slope of mPAP-LVCO $(5.8 \pm 2.4 \mathrm{vs} .2 .9 \pm$ $2.1 \mathrm{~mm} \mathrm{Hg} / \mathrm{L} / \mathrm{min} ; \mathrm{p}<0.0001)$. After adjustment for age and gender, exercise $\mathrm{LAP}(\beta=3.1 \pm 0.8 ; \mathrm{p}=0.001)$ and exercise PVR $(\beta=7.9 \pm 1.7 ; \mathrm{p}=0.0001)$ were independent determinants of exercise sPAP.

Conclusion: EIPH is frequent in SSc patients and is mainly related to both increased exercise LV filling pressure and exercise PVR.
\end{abstract}

(c) 2014 Elsevier Ireland Ltd. All rights reserved.

\section{Introduction}

Systemic sclerosis (SSc) is a rare and complex disease, characterized by an extensive vasculopathy associated with auto-antibodies and fibrosis, with a multifactorial etiology [1]. Pulmonary arterial hypertension $(\mathrm{PH})$, resulting from a pre-capillary mechanism, may be frequent in SSc with an approximated incidence of $8 \%$ to $13 \%$, leading to an increased morbidity and mortality [2]. Echocardiography is an accurate non-invasive tool for the daily-life screening of patients at risk of PH [3]. Exercise echocardiography assessment of the pulmonary circulatory

Abbreviations: CO, cardiac output; EIPH, exercise-induced pulmonary arterial hypertension; LAP, left atrial pressure; LV, left ventricular; mPAP, mean pulmonary arterial pressure; sPAP, systolic pulmonary arterial pressure; PH, pulmonary arterial pressure; PVR, pulmonary vascular resistance; SSc, systemic sclerosis.

* Corresponding authors at: Department of Cardiology, University Hospital, Université de Liège, CHU du Sart Tilman, 4000 Liège, Belgium. Tel.: + 32436671 94; fax: + 32 43667195.

E-mail addresses: lpierard@chu.ulg.ac.be (L.A. Pierard), plancellotti@chu.ulg.ac.be (P. Lancellotti). system has evolved over these last few years [4-6] and exerciseinduced PH (EIPH) has recently been suggested as a potential useful tool for the early identification of patients with SSc at risk of developing resting $\mathrm{PH}[7,8]$. It seems however, that the incidence of EIPH may overestimate the percentage of onset of resting PH during follow-up [9]. Some recent studies have underlined that the origin of EIPH in SSc could be secondary, not only to pulmonary vasculopathy, but also to myocardial [10] and/or pulmonary impairment [11,12]. Therefore, a post-capillary involvement in EIPH has been hypothesized in the literature $[13,14]$. Hitherto, the echocardiographic determinants of EIPH in SSc remain unclear. The present study sought to evaluate the incidence of EIPH and its determinants in patients with SSc.

\section{Methods}

We prospectively studied 68 consecutive patients from January 2008 to November 2012 with a diagnosis of SSc, followed in the rheumatology center of CHU Sart-Tilman in Liège. Five patients refused the study protocol. Exclusion criteria were: (1) inability to provide informed consent, (2) previous ischemic heart or valvular heart diseases and (3) inability to perform an exercise test. Eighteen patients were excluded, 15 due to 
poor echogenicity and unquantifiable SPAP secondary to severe thinness and/or pulmonary diseases, 1 for moderate mitral regurgitation and 2 for known coronary artery diseases. Informed consent was obtained from each patient. The study protocol conforms to the ethical guidelines of the 1975 Declaration of Helsinki as reflected in a priori approval by the institution's human research committee.

All patients underwent a comprehensive resting echocardiography using conventional method with a Vivid 9 ultrasound system (General Electric Healthcare, Little Chalfont, UK). Offline analysis was performed retrospectively using a customized software package (EchoPac). Left ventricular (LV) stroke volume were calculated as the difference between LV end-diastolic and systolic volumes assessed by the bi-apical Simpson disk method and LV ejection fraction was derived as the ratio stroke volume/LV end-diastolic volume. Cardiac output (CO) was obtained by multiplying LV SV and heart rate. Left atrial area was measured in apical 4-chamber view at end-systole by planimetry. Peak E- and peak A-wave velocities of the mitral inflow were measured with pulsed-wave Doppler. Doppler tissue imaging was applied for measurement of the $\mathrm{e}^{\prime}$ wave at the lateral mitral annulus aspect.

The following right ventricular echocardiographic parameters were assessed: right ventricular end-diastolic and end-systolic areas, right ventricular fractional area change, tricuspid annular plane systolic excursion from an automated M-mode applied on the right ventricular apical 4-chamber view and maximal systolic velocity of the tricuspid annulus derived from Doppler tissue imaging pulsed-wave at the lateral tricuspid annulus.

The systolic pulmonary arterial pressure (sPAP) was derived from the maximal velocity of tricuspid regurgitant jet according to the simplified Bernoulli equation and adding right atrial pressure, estimated from the dimension and collapsibility of the inferior vena cava, according to the American guidelines [15]. A peak value $>35 \mathrm{~mm} \mathrm{Hg}$ was considered to define resting $\mathrm{PH}$ [16]. At peak exercise, sPAP was derived from the maximal velocity of tricuspid regurgitant jet and adding $10 \mathrm{~mm} \mathrm{Hg}$ for the estimation of the right atrial pressure, as previously validated $[17,18]$. EIPH was defined as a $\mathrm{SPAP}>50 \mathrm{~mm} \mathrm{Hg}$ [19]. The mPAP was estimated by the Chemla formula: $\mathrm{mPAP}=0.61 \times \mathrm{sPAP}+2$. As previously described [20,21], left atrial pressure (LAP) was assessed by $1.9+1.24 \mathrm{LV} \mathrm{E} / \mathrm{e}^{\prime}$ and pulmonary vascular resistance (PVR) was estimated as the ratio between (mPAP-LAP) and LVCO, both at rest and at peak exercise. The slope of $\mathrm{MPAP} / \mathrm{LVCO}$ relationship was estimated as the ratio between changes (peak-rest value) in MPAP and changes in LVCO [19]. All measurements were performed according to the current European Association of Echocardiography/American Society of echocardiography recommendations [22].

A symptom-limited graded bicycle exercise was performed in a semi-supine position on a tilted table. After an initial workload of $25 \mathrm{~W}$ maintained for $2 \mathrm{~min}$, the workload was gradually increased by 25 W every 2 min. A 12-lead ECG was monitored continuously, and blood pressure was measured and rest and at each level of exercise. All patients presented normal tests, defined as the absence of the occurrence of 1) angina, 2) $\geq 2$-mm ST-segment depression compared with baseline level or 3) complex ventricular arrhythmias.

All patients underwent standard pulmonary function tests with assessment of total lung capacity, vital capacity, forced vital capacity, forced expiratory volume in $1 \mathrm{~s}$, ratio of forced expiratory volume in $1 \mathrm{~s}$ upon vital capacity and diffusing capacity of the lung for carbon monoxide.

Continuous variables are expressed as means \pm standard deviation; categorical variables are presented as numbers and percentages. Data comparisons were performed according to the presence or absence of EIPH using Student unpaired and paired $t$ test, $\chi^{2}$ test or Fischer exact test as appropriate. The relationships between exercise SPAP and other continuous variables (i.e., demographic data, resting and exercise echocardiographic data) were evaluated by simple linear regression. Independent determinant of exercise sPAP were obtained with the use of multiple linear regression. Values of $\mathrm{p}<0.05$ were considered statistically significant. All statistical analyses were performed with JMP version 10.0 .2

\section{Results}

\subsection{Population characteristics}

Fifteen patients were excluded due to unquantifiable SPAP, 1 for moderate mitral regurgitation and 2 for known coronary artery diseases. Among the remaining 45 patients, $47 \%$ developed EIPH ( $\mathrm{n}=$ 21). The sPAP increased significantly during exercise (from $25 \pm 7$ to $46 \pm 14 \mathrm{~mm} \mathrm{Hg} ; \mathrm{p}<0.0001)$. Patients with EIPH had higher resting $\operatorname{sPAP}(29 \pm 6$ vs. $21 \pm 5 \mathrm{~mm} \mathrm{Hg} ; \mathrm{p}<0.001)$, resting mPAP ( $20 \pm 4$ vs. $14 \pm 3 \mathrm{~mm} \mathrm{Hg} ; \mathrm{p}<0.0001$ ), exercise sPAP (58 \pm 9 vs. $36 \pm$ $8 \mathrm{~mm} \mathrm{Hg} ; \mathrm{p}<0.0001$, Fig. 1), exercise mPAP ( $37 \pm 6$ vs. $24 \pm$ $5 \mathrm{~mm} \mathrm{Hg} ; \mathrm{p}<0.0001)$ and slope of mPAP-LVCO relationship (5.8 \pm 2.4 vs. $2.9 \pm 2.1 \mathrm{~mm} \mathrm{Hg} / \mathrm{L} / \mathrm{min} ; \mathrm{p}<0.0001$, Fig. 2 ). In addition, all patients with resting $\mathrm{PH}(\mathrm{n}=3,7 \%)$ developed EIPH. Patients with EIPH were significantly older ( $62 \pm 12$ vs. $48 \pm 11$ years; $\mathrm{p}=0.0001)$, more frequently female ( $90 \%$ vs. $62 \%, \mathrm{p}=0.03$ ), had higher resting systolic blood pressure ( $139 \pm 22$ vs. $122 \pm 18 \mathrm{~mm} \mathrm{Hg} ; \mathrm{p}=0.03$ ) and resting pulse pressure ( $62 \pm 16$ vs. $50 \pm 11 \mathrm{~mm} \mathrm{Hg}$; $\mathrm{p}=0.02)$. There were

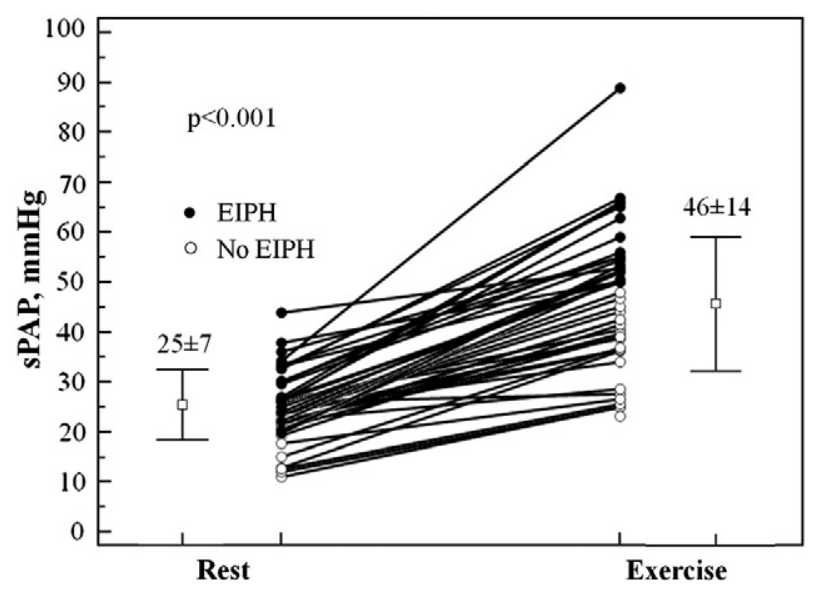

Fig. 1. Resting and exercise systolic pulmonary arterial pressure in the whole population. sPAP indicates systolic pulmonary arterial pressure; EIPH indicates exercise-induced pulmonary arterial hypertension.

no significant differences between the 2 groups regarding variables of lung function tests (Table 1).

\subsection{Resting and exercise echocardiography}

At rest (Table 2), patients of the EIPH group had significantly lower RV end-diastolic area $\left(12.5 \pm 3.1\right.$ vs. $\left.14.9 \pm 4.3 \mathrm{~cm}^{2} ; \mathrm{p}=0.04\right)$ higher resting PVR ( $2.6 \pm 0.8$ vs. $1.4 \pm 1.1$ vs. Woods unit; $\mathrm{p}=0.0004$ ), higher $\mathrm{LVE} / \mathrm{e}^{\prime}$ ratio $(6.8 \pm 1.8$ versus $5.5 \pm 1.8 ; \mathrm{p}=0.03)$, as a result of a lower $\mathrm{e}^{\prime}(11 \pm 3$ versus $13 \pm 3 \mathrm{~cm} / \mathrm{s} ; \mathrm{p}=0.03)$, and higher LAP $(10.3 \pm 0.5$ vs. $8.8 \pm 2.3 \mathrm{~mm} \mathrm{Hg}, \mathrm{p}=0.03$ ), as compared to patients of the no EIPH group. During exercise, EIPH group exhibited (Table 3 ) higher LV E/e $\mathrm{e}^{\prime}$ ratio resulting from lower value of $\mathrm{e}^{\prime}$ (respectively: $9.2 \pm 1.8$ vs. $5.7 \pm 1.4 ; \mathrm{p}<0.0001$ and $13 \pm 1$ vs. $17 \pm 4 \mathrm{~cm} / \mathrm{s} ; \mathrm{p}=0.01)$, higher LAP $(13.3 \pm 2.3$ vs. $9 \pm 0.5 \mathrm{~mm} \mathrm{Hg} ; \mathrm{p}<0.0001)$. The LVCO significantly increased from rest to exercise in all patients ( $3.7 \pm 0.9 \mathrm{vs}$. $7.2 \pm 2.0 \mathrm{~L} / \mathrm{min} ; \mathrm{p}<0.001$, Fig. 3$)$, but in a lower extent in the EIPH group ( $4.2 \pm 1.9$ vs. $3.0 \pm 1.3 \mathrm{~L} / \mathrm{min} ; \mathrm{p}=0.04)$ and patients of this group had a significantly lower resting LV end-systolic volume ( $25 \pm$ 6 vs. $31 \pm 11 \mathrm{~mL} ; \mathrm{p}=0.04)$ and exercise-induced changes in LV enddiastolic diameter in the EIPH group $(-8 \pm 25$ vs. $24 \pm 46 \mathrm{~mm}$; $\mathrm{p}=$ 0.002). Exercise PVR was significantly higher in patients with EIPH ( $3.6 \pm 0.7$ vs. $2.1 \pm 0.9$ Woods units; $\mathrm{p}<0.0001)$.

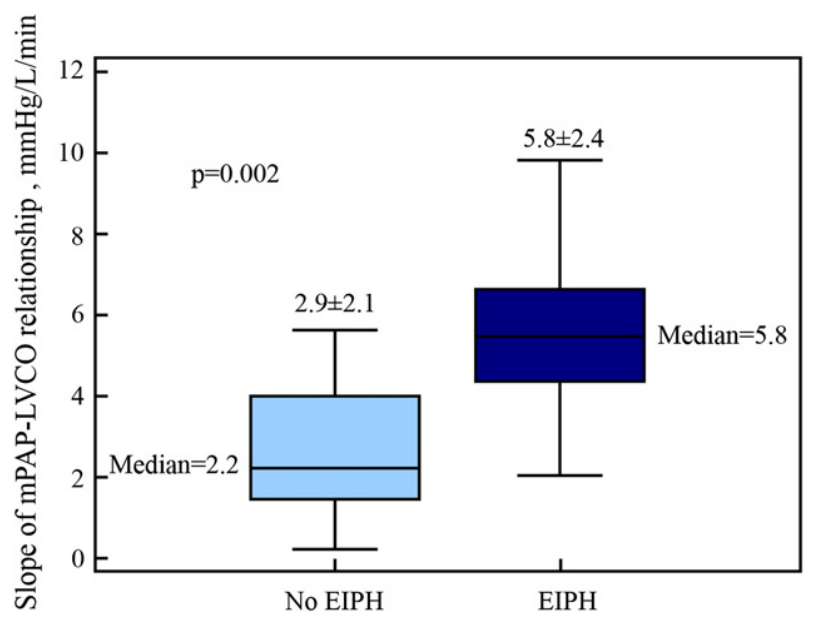

Fig. 2. Comparison of the slope of mean pulmonary arterial pressure and left ventricular relationships between the two groups. Slope of mPAP-LVCO indicates the ratio between change in mean pulmonary arterial pressure and change in left ventricular cardiac output; EIPH indicates exercise-induced pulmonary arterial hypertension. 
Table 1

Demographic, clinical, and exercise data.

\begin{tabular}{|c|c|c|c|c|}
\hline Variables & Whole cohort $(n=45)$ & No EIPH $(\mathrm{n}=24,53 \%)$ & $\mathrm{EIPH}(\mathrm{n}=21,47 \%)$ & $\mathrm{p}$ \\
\hline \multicolumn{5}{|l|}{ Demographic and clinical data } \\
\hline Age, years & $54 \pm 13$ & $48 \pm 11$ & $62 \pm 12$ & 0.0001 \\
\hline Female gender, n (\%) & $34(76)$ & $15(62)$ & $19(90)$ & 0.03 \\
\hline Body mass index, $\mathrm{kg} / \mathrm{m}^{2}$ & $24 \pm 5$ & $24 \pm 5$ & $24 \pm 5$ & 1 \\
\hline Heart rate, bpm & $73 \pm 13$ & $75 \pm 13$ & $72 \pm 13$ & 0.38 \\
\hline Systolic arterial pressure, $\mathrm{mm} \mathrm{Hg}$ & $131 \pm 22$ & $122 \pm 18$ & $139 \pm 22$ & 0.03 \\
\hline Diastolic arterial pressure, $\mathrm{mm} \mathrm{Hg}$ & $74 \pm 11$ & $72 \pm 11$ & $77 \pm 10$ & 0.19 \\
\hline Pulse pressure, $\mathrm{mm} \mathrm{Hg}$ & $56 \pm 15$ & $50 \pm 11$ & $62 \pm 16$ & 0.02 \\
\hline \multicolumn{5}{|l|}{ Pulmonary function } \\
\hline Total lung capacity, \% predicted & $93 \pm 21$ & $94 \pm 22$ & $92 \pm 21$ & 0.75 \\
\hline Vital capacity, \% predicted & $104 \pm 24$ & $110 \pm 25$ & $99 \pm 23$ & 0.18 \\
\hline Force vital capacity, $\%$ predicted & $101 \pm 21$ & $104 \pm 21$ & $99 \pm 22$ & 0.42 \\
\hline FEV1, \% predicted & $95 \pm 21$ & $97 \pm 21$ & $94 \pm 21$ & 0.69 \\
\hline FEV1/vital capacity, \% predicted & $99 \pm 12$ & $98 \pm 11$ & $100 \pm 18$ & 0.56 \\
\hline DLCO, \% predicted & $65 \pm 14$ & $67 \pm 14$ & $63 \pm 15$ & 0.45 \\
\hline \multicolumn{5}{|l|}{ Risk factors } \\
\hline Systemic hypertension, n (\%) & $5(11)$ & $1(4)$ & $4(19)$ & 0.12 \\
\hline Hypercholesterolemia, n (\%) & $9(20)$ & $2(9)$ & $7(33)$ & 0.04 \\
\hline Diabetes mellitus, $\mathrm{n}(\%)$ & 0 & 0 & 0 & NA \\
\hline Active smoker, n (\%) & $9(20)$ & $8(35)$ & $1(5)$ & 0.01 \\
\hline Family history of CV disease, $\mathrm{n}(\%)$ & 0 & 0 & 0 & NA \\
\hline \multicolumn{5}{|l|}{ Medication } \\
\hline ACE inhibitors, $n(\%)$ & $4(10)$ & $1(45)$ & $3(15)$ & 0.29 \\
\hline$\beta$-Blockers, n (\%) & $4(10)$ & $0(0)$ & $4(25)$ & NA \\
\hline Diuretic, n (\%) & $2(5)$ & $1(5)$ & $1(5)$ & 1 \\
\hline Calcium channel blockers, n (\%) & $22(55)$ & $13(65)$ & $9(45)$ & 0.20 \\
\hline Corticoids, n (\%) & $12(30)$ & $5(25)$ & $7(35)$ & 0.49 \\
\hline Immunosuppressors, $\mathrm{n}(\%)$ & $7(17.5)$ & $4(20)$ & $3(15)$ & 0.68 \\
\hline \multicolumn{5}{|l|}{ Exercise data } \\
\hline Workload, W & $74 \pm 31$ & $86 \pm 31$ & $65 \pm 28$ & 0.052 \\
\hline Duration of exercise, min & $4.7 \pm 1.3$ & $4.6 \pm 1.3$ & $5 \pm 1.3$ & 0.30 \\
\hline Heart rate, bpm & $118 \pm 18$ & $118 \pm 17$ & $117 \pm 19$ & 0.20 \\
\hline Systolic arterial pressure, $\mathrm{mm} \mathrm{Hg}$ & $169 \pm 22$ & $162 \pm 24$ & $175 \pm 16$ & 0.11 \\
\hline Diastolic arterial pressure, $\mathrm{mm} \mathrm{Hg}$ & $82 \pm 11$ & $79 \pm 9$ & $85 \pm 13$ & 0.19 \\
\hline Pulse pressure, $\mathrm{mm} \mathrm{Hg}$ & $86 \pm 15$ & $83 \pm 18$ & $90 \pm 11$ & 0.17 \\
\hline
\end{tabular}

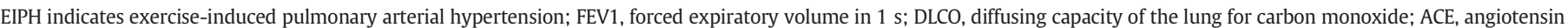
converting enzyme; and CV, cardio-vascular.

\subsection{Determinants of exercise sPAP}

A significant correlation was found between exercise sPAP and exercise LAP $\left(r^{2}=0.61, p<0.0001\right.$, Fig. 3), exercise-induced changes in LV enddiastolic diameter $\left(\mathrm{r}^{2}=0.26, \mathrm{p}=0.0013\right)$ and age $\left(\mathrm{r}^{2}=0.20, \mathrm{p}=\right.$ 0.002 ) (Table 4). Exercise LAP was correlated with exercise LA area $\left(r^{2}=0.32, p=0.02\right.$, Fig. 4). Exercise PVR was significantly associated with exercise $\operatorname{sPAP}\left(r^{2}=0.57 ; p=0.0004\right.$, Fig. 5). After adjustment for age and gender, resting PVR $(\beta=6.2 \pm 1.5 ; \mathrm{p}=$ $0.0001)$, exercise LAP $(\beta=3.1 \pm 0.8 ; p=0.001)$ and exercise PVR $(\beta=7.9 \pm 1.7 ; p=0.0001)$ remained independently associated with exercise sPAP. In multiple linear regression (model including age and gender, exercise LAP and exercise PVR), female gender ( $\beta=6.8 \pm$ $2.8 ; \mathrm{p}=0.03)$, exercise LAP $(\beta=1.9 \pm 0.6 ; \mathrm{p}=0.005)$ and exercise PVR $(\beta=6.8 \pm 1.4 ; \mathrm{p}<0.001)$ remained significantly associated with exercise sPAP.

\section{Discussion}

The present study shows that (1) EIPH is common in patients with SSc (47\%), (2) main mechanisms explaining EIPH are increased estimated exercise LV filling pressure and increased PVR and (3) taking into account the main factors generally considered as influencing sPAP (e.g. age, CO or pulmonary disease), the relationship between estimated exercise LV filling pressure, exercise PVR and exercise SPAP remains significant.

\subsection{Incidence of EIPH in SSC}

EIPH is not rare in SSc, even in young patients. Alkotob et al. [7] found an incidence of $46 \%$. More recently, Gargani et al. [19] have shown an incidence of $42 \%$ in a population of 164 patients with SSc. Of note, mean age of the population in these studies was relatively similar. Even if the criterion used to define the presence of EIPH (sPAP increase $\geq 20 \mathrm{~mm} \mathrm{Hg}$ [23], or exercise sPAP $>50 \mathrm{~mm} \mathrm{Hg}$ [19]), the incidence of EIPH reported remained almost similar. Our results confirm and extend these previous studies $[7,23,19]$ showing a rate of $47 \%$ of EIPH in a population with a mean age of 54 -year old.

\subsection{Mechanisms of EIPH in SSC}

In our study, main mechanisms explaining the difference in exercise sPAP between the 2 groups are 1 ) higher exercise estimated LV filling pressure and 2) higher exercise PVR.

Thus, our results support the presence of a sub-clinical LV diastolic dysfunction [24]. The presence and prognostic value of sub-clinical diastolic dysfunction has already been demonstrated in various diseases $[25,26]$. Studies based on right heart catheterization have also reported a high rate of exercise diastolic impairment in SSc. However, the mechanisms involved in this dysfunction were not clearly identified [13] and several mechanisms have been suggested. In SSc, diastolic dysfunction depends not only on classical risk factors such as age, hypertension or diabetes but also on other specific factors such as the chronicity of disease [27] and micro-vascular impairment [28].

We found a low incidence of diabetes and hypertension. However, this is not in contradiction with the increase in exercise LV filling pressure. Our population was relatively "young" ( $54 \pm 13$ years) and included a high percentage of female gender (76\%), partly explaining this fact. These results are consistent with other studies [14,19] with relatively similar population (mean age from 54 to 58 years and male gender from 7 to $9 \%$ ). The authors also found low incidence of diabetes 
Table 2

Resting echocardiographic data.

\begin{tabular}{|c|c|c|c|c|}
\hline Variables & Whole cohort $(n=45)$ & No EIPH $(n=24,53 \%)$ & $\operatorname{EIPH}(n=21,47 \%)$ & $\mathrm{p}$ \\
\hline \multicolumn{5}{|l|}{ Resting LV echocardiographic data } \\
\hline LV end-diastolic volume, mL & $79 \pm 19$ & $84 \pm 24$ & $74 \pm 12$ & 0.10 \\
\hline LV end-systolic volume, mL & $28 \pm 9$ & $31 \pm 11$ & $25 \pm 6$ & 0.04 \\
\hline LV stroke volume, $\mathrm{mL}$ & $51 \pm 12$ & $53 \pm 15$ & $50 \pm 9$ & 0.45 \\
\hline LVEF Simpson, \% & $65 \pm 6$ & $63 \pm 4$ & $66 \pm 7$ & 0.12 \\
\hline $\mathrm{CO}, \mathrm{L} / \mathrm{min}$ & $3.7 \pm 0.9$ & $3.9 \pm 1.1$ & $3.5 \pm 0.7$ & 0.19 \\
\hline $\mathrm{E}, \mathrm{m} / \mathrm{s}$ & $0.7 \pm 0.1$ & $0.7 \pm 0.1$ & $0.7 \pm 0.1$ & 0.89 \\
\hline E deceleration time, ms & $178 \pm 37$ & $172 \pm 30$ & $187 \pm 43$ & 0.15 \\
\hline $\mathrm{A}^{\prime}, \mathrm{m} / \mathrm{s}$ & $0.7 \pm 0.2$ & $0.6 \pm 0.2$ & $0.7 \pm 0.2$ & 0.32 \\
\hline $\mathrm{E} / \mathrm{A}^{\prime}$ ratio & $1.1 \pm 0.5$ & $1.1 \pm 0.4$ & $1.1 \pm 0.6$ & 0.82 \\
\hline $\mathrm{e}^{\prime}, \mathrm{cm} / \mathrm{s}$ & $12 \pm 3$ & $13 \pm 3$ & $11 \pm 3$ & 0.03 \\
\hline $\mathrm{E} / \mathrm{e}^{\prime}$ ratio & $6.0 \pm 1.9$ & $5.5 \pm 1.8$ & $6.8 \pm 1.8$ & 0.03 \\
\hline LAP, mm Hg & $9.4 \pm 2.4$ & $8.8 \pm 2.3$ & $10.3 \pm 2.2$ & 0.03 \\
\hline LV eccentricity index & $92 \pm 12$ & $90 \pm 11$ & $94 \pm 13$ & 0.31 \\
\hline \multicolumn{5}{|l|}{ Resting RV echocardiographic data } \\
\hline $\mathrm{RV} / \mathrm{LV}$ ratio & $74.4 \pm 8.6$ & $74.5 \pm 9.3$ & $74.3 \pm 8.1$ & 0.94 \\
\hline RV end-diastolic area, $\mathrm{cm}^{2}$ & $13.7 \pm 3.9$ & $14.9 \pm 4.3$ & $12.5 \pm 3.1$ & 0.04 \\
\hline RV end-systolic area, $\mathrm{cm}^{2}$ & $7.2 \pm 2.5$ & $7.9 \pm 2.4$ & $6.5 \pm 2.4$ & 0.09 \\
\hline RVFAC, \% & $47.8 \pm 8.8$ & $47.3 \pm 7.4$ & $48.2 \pm 10.2$ & 0.75 \\
\hline TAPSE, $\mathrm{mm}$ & $23 \pm 5$ & $23 \pm 5$ & $23 \pm 5$ & 0.72 \\
\hline $\mathrm{s}^{\prime}, \mathrm{cm} / \mathrm{s}$ & $12.2 \pm 2.7$ & $12.1 \pm 3.1$ & $12.4 \pm 2.2$ & 0.68 \\
\hline ICV $\max , \mathrm{cm} / \mathrm{s}$ & $11.7 \pm 4.0$ & $11.6 \pm 4.1$ & $11.9 \pm 4.0$ & 0.79 \\
\hline IVRT, ms & $46 \pm 23$ & $47 \pm 5$ & $44 \pm 5$ & 0.66 \\
\hline MPI & $0.35 \pm 0.14$ & $0.34 \pm 0.10$ & $0.36 \pm 0.17$ & 0.61 \\
\hline Pulmonary acceleration time, ms & $138 \pm 34$ & $141 \pm 35$ & $133 \pm 33$ & 0.47 \\
\hline Resting PVR, WU & $1.9 \pm .12$ & $1.4 \pm 1.1$ & $2.6 \pm 0.8$ & 0.0004 \\
\hline $\mathrm{sPAP}, \mathrm{mm} \mathrm{Hg}$ & $25 \pm 7$ & $21 \pm 5$ & $29 \pm 6$ & $<0.0001$ \\
\hline mPAP, mm Hg & $17 \pm 4$ & $14 \pm 3$ & $20 \pm 4$ & $<0.0001$ \\
\hline \multicolumn{5}{|l|}{ Resting atrial area } \\
\hline $\mathrm{LA}, \mathrm{cm}^{2}$ & $14 \pm 4$ & $14 \pm 3$ & $15 \pm 5$ & 0.24 \\
\hline $\mathrm{RA}, \mathrm{cm}^{2}$ & $12 \pm 4$ & $12 \pm 3$ & $12 \pm 5$ & 0.96 \\
\hline
\end{tabular}

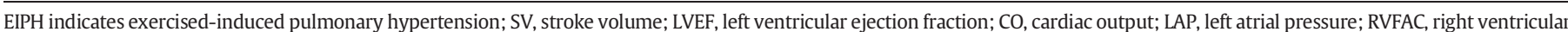

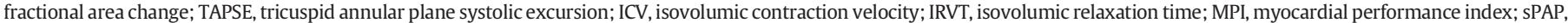
systolic pulmonary arterial pressure; mPAP, mean pulmonary arterial pressure; PVR, pulmonary vascular resistance; WU, Woods unit, RA, right atrial; and LA, left atrial.

(0 to $4 \%$ ) and of hypertension (24 to $29 \%$ ). In patients with SSc, exercise sub-clinical diastolic dysfunction could also be the result of other mechanisms.

First, microvascular ischemia [29] may increase LV stiffness and reduce LV compliance, leading to an increased in estimated LV filling pressure. This phenomenon was previously reported by Bulkley et al. [30] who highlighted diffuse patchy fibrosis on histological examinations, with contraction band of necrosis unrelated to epicardial coronary artery stenosis. In addition, James et al. [31] revealed concentric intimal hypertrophy associated with fibrinoid necrosis of intramural coronary arteries. Some authors demonstrated microvascular ischemia by single photon emission computed tomography study of myocardial perfusion. Myocardial scintigraphy demonstrated evidence of reversible ischemia together with irreversible lesions and showed inducible of coronary vasospasm by cold pressor provocation, suggesting both myocardial ischemia and fibrosis [32]. Steen et al. [33] demonstrated that exerciseinduced perfusion defects seen by scintigraphy predict onset of cardiac diseases and death. Several studies emphasized the key role of microvascular ischemia due to vasospasm and demonstrated the beneficial effect of vasodilator agents on perfusion abnormalities [34]. Vignaux et al. [35] demonstrated the link between micro-vascular ischemia and systolic and/or diastolic function. They showed an increase in systolic and diastolic function, as assessed with strain rate, after administration of nifedipine in patients with SSc. At the same time, they showed an increase in perfusion, as assessed by magnetic resonance imaging, suggesting that increase in myocardial perfusion was the main determinant of the increase in systolic and diastolic function in this population. All these studies highlighted that micro-vascular function has a key role in the pathophysiology of systolic and diastolic myocardial dysfunction in SSc.

Of note, the absence of kinetic disorders, chest pain or ECG changes in our study does not exclude this mechanism. In fact, considering the ischemic cascade [36], diastolic dysfunction appears early after perfusion abnormalities and before systolic dysfunction, ECG changes and chest pain.

The second mechanism could be related to a poor exercise adaptation of the systemic vascular system resulting to an excessive arterial stiffness. Several studies have highlighted arterial systemic stiffness in SSc [28-30], with heterogeneous impairment of large-vessels [37]. Cheng et al. have recently confirmed increased stiffness in systemic vascular system of SSc patients with a reduction of elastic properties of carotid artery [38]. Of note, in our study, despite higher pulse pressure at rest in the EIPH group, there was no significant difference during exercise. Pulse pressure depends on stroke volume and arterial compliance. We did not find significant differences between the 2 groups in terms of stroke volume, suggesting a lower systemic vascular compliance in the EIPH group. Interestingly, Cheng et al. showed increased stiffness in elastic arterial (carotid) and not in muscular artery (femoral) suggesting different elastic properties depending on the type of artery. Moreover, Liu et al. [37] demonstrated preferential macro-vasculopathy on aorta (elastic artery), possibly explaining why pulse pressure measured at the upper arm (muscular artery) was not different during exercise in our population.

We also demonstrated that there might be pulmonary vascular impairment in this population, with higher exercise PVR in the EIPH group. This phenomenon is well known in SSc patients and may explain the onset of resting PH. Pulmonary arterial stiffness is due to pulmonary vascular remodeling, in situ thrombosis but also infiltration of inflammatory cells leading to endothelial proliferation. Even in the subset of patients with no EIPH, we found an abnormal increase of PVR ( $>3$ Wood units) in $21 \%$ of them. This result is in line with previous study from Gargani et al. [19] demonstrating a change of $3.1 \pm$ $2.7 \mathrm{~mm} \mathrm{Hg} / \mathrm{L} / \mathrm{min}$ in the subset of patients with exercise sPAP $<50 \mathrm{~mm} \mathrm{Hg}$. The authors also analyzed a sub-group of patients with 
Table 3

Exercise echocardiographic data.

\begin{tabular}{|c|c|c|c|c|}
\hline Variables & Whole cohort $(n=45)$ & No EIPH ( $\mathrm{n}=24,53 \%)$ & $\operatorname{EIPH}(n=21,47 \%)$ & $\mathrm{p}$ \\
\hline \multicolumn{5}{|l|}{ Exercise LV echocardiographic data } \\
\hline LV end-diastolic volume, mL & $88 \pm 22$ & $93 \pm 24$ & $82 \pm 16$ & 0.13 \\
\hline LV end-systolic volume, mL & $27 \pm 9$ & $29 \pm 10$ & $25 \pm 6$ & 0.18 \\
\hline LV stroke volume, $\mathrm{mL}$ & $61 \pm 16$ & $64 \pm 17$ & $57 \pm 13$ & 0.14 \\
\hline LVEF Simpson, \% & $70 \pm 6$ & $70 \pm 6$ & $70 \pm 5$ & 1.00 \\
\hline $\mathrm{CO}, \mathrm{L} / \mathrm{mn}$ & $7.2 \pm 2.0$ & $7.7 \pm 2.4$ & $6.5 \pm 1.2$ & 0.08 \\
\hline Delta $\mathrm{CO}, \mathrm{L} / \mathrm{mn}$ & $3.5 \pm 1.6$ & $4 \pm 1.7$ & $3 \pm 1.3$ & 0.10 \\
\hline $\mathrm{E}, \mathrm{m} / \mathrm{s}$ & $1.0 \pm 0.2$ & $1.0 \pm 0.2$ & $1.0 \pm 0.2$ & 0.52 \\
\hline E dec time, ms & $121 \pm 39$ & $120 \pm 41$ & $112 \pm 37$ & 0.89 \\
\hline $\mathrm{A}^{\prime}, \mathrm{m} / \mathrm{s}$ & $0.9 \pm 0.2$ & $0.9 \pm 0.1$ & $1.0 \pm 0.2$ & 0.12 \\
\hline $\mathrm{E} / \mathrm{A}^{\prime}$ ratio & $1.1 \pm 0.2$ & $1.1 \pm 0.2$ & $1.0 \pm 0.1$ & 0.54 \\
\hline $\mathrm{e}^{\prime}, \mathrm{cm} / \mathrm{s}$ & $16 \pm 4$ & $17 \pm 4$ & $13 \pm 1$ & 0.01 \\
\hline $\mathrm{E} / \mathrm{e}^{\prime}$ ratio & $6.8 \pm 2.2$ & $5.7 \pm 1.4$ & $9.2 \pm 1.8$ & $<0.0001$ \\
\hline LAP, mm Hg & $10.3 \pm 2.8$ & $9 \pm 1.7$ & $13.3 \pm 2.3$ & $<0.0001$ \\
\hline Changes in LAP, $\mathrm{mm} \mathrm{Hg}$ & $1.4 \pm 2.2$ & $0.8 \pm 2.2$ & $3.1 \pm 1.4$ & 0.03 \\
\hline \multicolumn{5}{|l|}{ Exercise RV echocardiographic data } \\
\hline RV end-diastolic area, $\mathrm{cm}^{2}$ & $12.1 \pm 3.0$ & $12.6 \pm 3.1$ & $11.6 \pm 2.9$ & 0.41 \\
\hline RV end-systolic area, $\mathrm{cm}^{2}$ & $5.7 \pm 1.6$ & $6.1 \pm 2.0$ & $5.2 \pm 1.0$ & 0.15 \\
\hline RVFAC, \% & $52.6 \pm 10.0$ & $51.4 \pm 10.4$ & $53.6 \pm 10.1$ & 0.60 \\
\hline LV end-diastolic diameter, mm & $39 \pm 5$ & $42 \pm 5$ & $36 \pm 3$ & 0.0005 \\
\hline $\mathrm{RV} / \mathrm{LV}$ ratio & $72 \pm 12$ & $67 \pm 10$ & $77 \pm 13$ & 0.03 \\
\hline TAPSE, mm & $27 \pm 5$ & $26 \pm 3$ & $28 \pm 7$ & 0.20 \\
\hline $\mathrm{s}^{\prime}, \mathrm{cm} / \mathrm{s}$ & $16.4 \pm 3.7$ & $16.9 \pm 4.2$ & $15.8 \pm 2.9$ & 0.44 \\
\hline ICV $\max , \mathrm{cm} / \mathrm{s}$ & $15.5 \pm 4.7$ & $15.3 \pm 5.9$ & $15.9 \pm 2.7$ & 0.78 \\
\hline IVRT, ms & $39 \pm 17$ & $37 \pm 5$ & $40 \pm 5$ & 0.64 \\
\hline $\mathrm{sPAP}, \mathrm{mm} \mathrm{Hg}$ & $46 \pm 14$ & $36 \pm 8$ & $58 \pm 9$ & $<0.0001$ \\
\hline mPAP, mm Hg & $30 \pm 8$ & $24 \pm 5$ & $37 \pm 6$ & $<0.0001$ \\
\hline Changes in mPAP, $\mathrm{mm} \mathrm{Hg}$ & $13 \pm 6$ & $10 \pm 4$ & $17 \pm 6$ & $<0.0001$ \\
\hline Slope of mPAP-LVCO, $\mathrm{mm} \mathrm{Hg} / \mathrm{L} / \mathrm{min}$ & $4.2 \pm 2.6$ & $2.9 \pm 2.1$ & $5.8 \pm 2.4$ & 0.002 \\
\hline PVR, WU & $2.5 \pm 1.1$ & $2.1 \pm 0.9$ & $3.6 \pm 0.7$ & 0.01 \\
\hline \multicolumn{5}{|l|}{ Exercise atrial area } \\
\hline $\mathrm{LA}$ area, $\mathrm{cm}^{2}$ & $14 \pm 5$ & $14 \pm 4$ & $15 \pm 6$ & 0.36 \\
\hline $\mathrm{RA}$ area, $\mathrm{cm}^{2}$ & $13 \pm 4$ & $12 \pm 3$ & $13 \pm 6$ & 0.50 \\
\hline
\end{tabular}

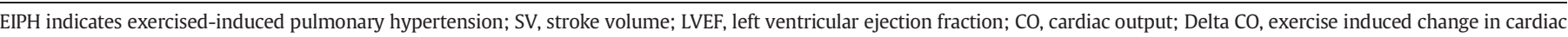

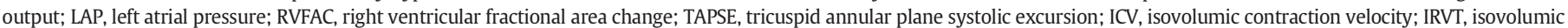

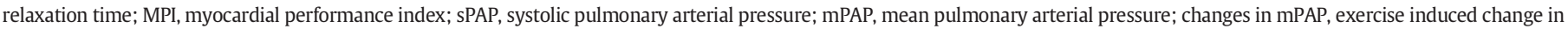
mPAP; slope of mPAP, ratio between changes in mPAP and changes in LVCO; PVR, pulmonary vascular resistance; RA, right atrial; and LA, left atrial.

age $<50$ year-old and showed that even in this low risk patients, an abnormal increase of PVR at peak exercise was present (approximately around $25 \%$, from 1.6 at rest to 2.0 Wood units at peak exercise), as compared to controls. These results may suggest that even in subgroup of patients with low risk factors of $\mathrm{PH}$, pulmonary microvascular impairment may be present.

Finally, abnormal increase in SPAP in SSc may result from both preand post-capillary involvement as demonstrated by the multiple linear regression analysis.

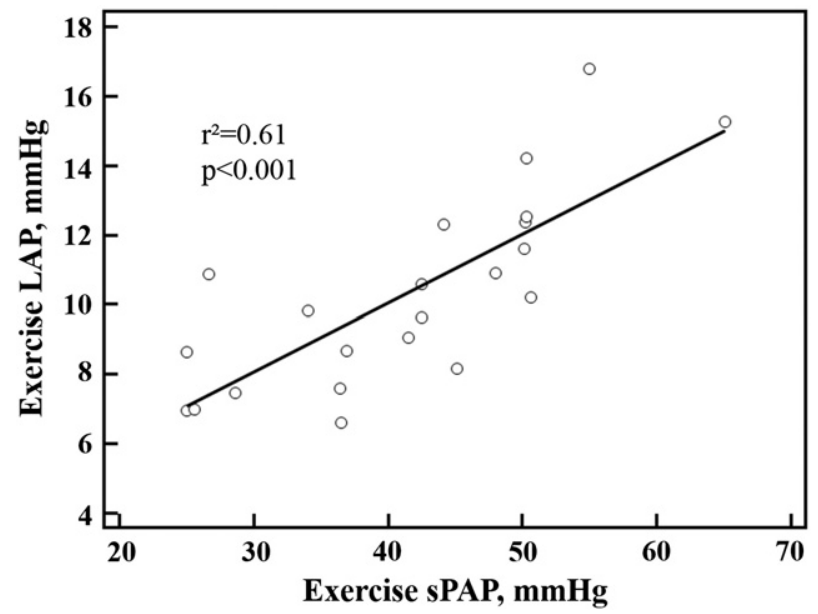

Fig. 3. Relationship between exercise systolic pulmonary arterial pressure and exercise left atrial pressure. sPAP indicates systolic pulmonary arterial pressure; LAP indicates left atrial pressure.
Although populations, definition of exercise-induced pulmonary hypertension and methods of investigations were not similar, the coexistence of both mechanisms has already been demonstrated in several studies. Hager et al. [13] performed exercise right heart catheterization in 173 patients with resting $\mathrm{SPAP}<40 \mathrm{~mm} \mathrm{Hg}$, exercise sPAP $>40 \mathrm{~mm} \mathrm{Hg}$ and shortness of breath. EIPH was defined as the ratio between changes in mPAP and changes in $\mathrm{CO}>2$ in patients $<50$ year old and $>3$ in older ones. Pre- or post-capillary involvements were defined depending on exercise trans-pulmonary gradient, pulmonary capillary wedge pressure values and changes in PVR. Of 53 patients with EIPH, 6 had a pre-capillary involvement (11\%) and 47 had exercise diastolic dysfunction (89\%), suggesting a complex pathophysiology. In another study, Ciurzyński et al. [14] performed resting and postexercise assessment of sPAP in 85 patients with SSc. Twenty patients

Table 4

Significant relationship between clinical and echocardiographic data and exercise sPAP.

\begin{tabular}{llc}
\hline Variables & $\mathrm{r}^{2}$ & $\mathrm{p}$ \\
\hline Age & 0.20 & 0.002 \\
Resting PVR & 0.39 & $<0.0001$ \\
Exercise RV end-diastolic diameter & 0.10 & 0.06 \\
Exercise LV end-systolic diameter & 0.26 & 0.001 \\
Exercise RV/LV diameter & 0.17 & 0.02 \\
Exercise LV e', m/s & 0.37 & 0.001 \\
Exercise LAP & 0.61 & $<0.0001$ \\
Exercise PVR & 0.57 & 0.0004 \\
Exercise-induced change in RV end-diastolic area & 0.17 & 0.03 \\
Exercise-induced change in LV end-diastolic diameter & 0.20 & 0.006 \\
Exercise-induced change in RV/LV ratio & 0.17 & 0.02 \\
\hline
\end{tabular}

PVR indicates pulmonary vascular resistance; RV, right ventricular; LV, left ventricular; and LAP, left atrial pressure. 


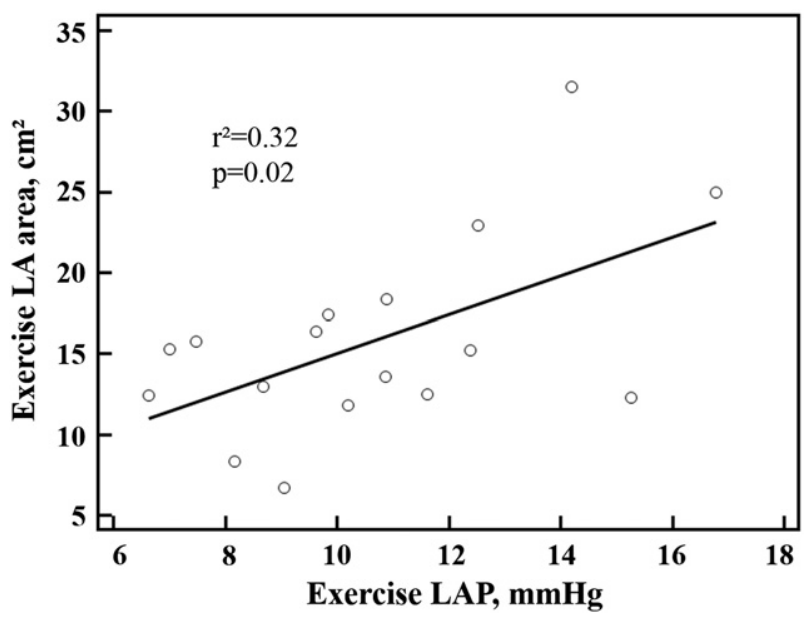

Fig. 4. Relationship between exercise left atrial pressure and exercise left atrial area. LAP indicates left atrial pressure; LA indicates left atrial.

with an abnormal increase in $\operatorname{SPAP}(>20 \mathrm{~mm} \mathrm{Hg})$ were referred to right heart catheterization. Twelve patients had EIPH (mPAP $>30 \mathrm{~mm} \mathrm{Hg}$ ) with elevated exercise pulmonary capillary wedge pressure ( $>20 \mathrm{~mm} \mathrm{Hg}$ ) and 5 with pre-capillary participation. The authors concluded that abnormal increase of PAP in SSc patients was more commonly caused by LV diastolic dysfunction than pulmonary arterial vasculopathy.

Our results are consistent with these previous studies and suggest that EIPH in SSc may result from both pre- and post-capillary mechanisms due to global myocardial and pulmonary microvascular impairment.

\subsection{Factors influencing sPAP}

The sPAP is strongly influenced by several factors such as age, pulmonary disease and CO. Influence of age on SPAP is demonstrated in several right heart catheterization studies in healthy subjects [39]. More recently, this relationship has been confirmed in non-invasive studies. Argiento et al. [20] have shown significantly higher mPAP both at rest and at peak exercise in subjects older than 50 year old (respectively: 17,3 vs. $14.9,2.5$; p $<0.05$ and $35.9,5.3$ vs. 32.4, $7.4 \mathrm{~mm} \mathrm{Hg} ; \mathrm{p}<0.05)$. Similar results were found by Mahjoub et al. [40] in an exercise echocardiography study with SPAP at peak exercise

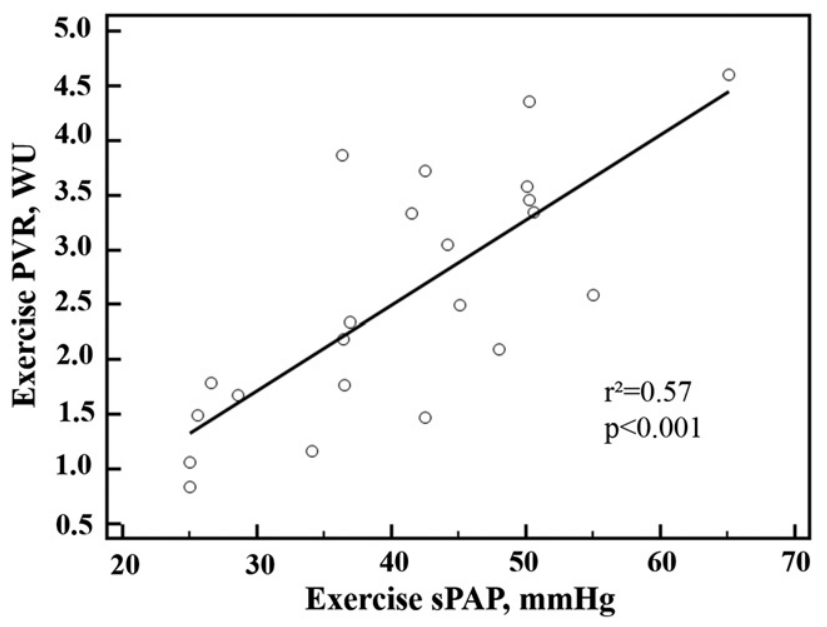

Fig. 5. Relationship between exercise systolic pulmonary arterial pressure and exercise pulmonary vascular resistance. SPAP indicates systolic pulmonary arterial pressure; PVR indicates pulmonary vascular resistance, WU, indicates Woods unit. of $45 \pm 7 \mathrm{~mm} \mathrm{Hg}$ for <30-year old subjects to $58 \pm 7 \mathrm{~mm} \mathrm{Hg}$ for $>70$-year old subjects. In our study, the EIPH group was significantly older but after adjustment for age, exercise $\mathrm{E} / \mathrm{e}^{\prime}$ ratio remained associated with SPAP.

The sPAP is also related to the CO. In healthy subjects, there is a linear relationship between $\mathrm{CO}$, age and SPAP [4]. We did not find significant difference in LVCO between the 2 groups, with even a trend toward lower exercise LVCO in the EIPH group and a lower exercise-induced change in LVCO. Furthermore, the slope of mPAP-LVCO relationship remained significantly higher in this group.

Finally, pulmonary diseases may influence sPAP. There is a close relationship between pulmonary function and pulmonary circulation and especially in SSc with interstitial lung diseases [41]. No significant differences between the 2 groups regarding variables of lung function tests were found in the present study.

\subsection{Limitations of the study}

The main limitation of our study is the relative small size of the population. However, it reflects the low incidence of SSc. For instance, the absence of relationship between workload or pulmonary acceleration time (Tables 1 and 2) and EIPH could mainly be due to a type II error.

Small LV volumes reported could be related to foreshortening views and may also explain the low LVCO reported. However this underestimation affects the whole population. Consequently, this limitation does not influence the reliability of the main results of this study, which is the demonstration that estimated LV filling pressure during exercise is an independent determinant of EIPH.

As previously described $[20,21]$ and underlined by several recent reviews [4-6], the slope of the MPAP-LVCO relationship is probably the best way to demonstrate an abnormal increase in PAP during exercise and therefore for the diagnosis EIPH. However, duration of exercise in our population was really short and we rather focused on LV systolic and diastolic function. In addition, it has been demonstrated that for a LVCO less than $10 \mathrm{~L} / \mathrm{min}$, the upper limit of normal value of mPAP is $34 \mathrm{~mm} \mathrm{Hg}$ [4], corresponding to a sPAP of $55 \mathrm{~mm} \mathrm{Hg}$. In our cohort, all but 4 patients in the no EIPH group exhibited a peak exercise LVCO $>10 \mathrm{~L} / \mathrm{min}$. As echocardiography assessment of sPAP most of the time underestimates this value, a cut-off value of $50 \mathrm{~mm} \mathrm{Hg}$ (security margin of $5 \mathrm{~mm} \mathrm{Hg}$ ) is acceptable in this kind of population. However, we made an estimation of the slope of MPAP-LVCO relationship, using the ratio between changes in MPAP and changes in LVCO (peak-rest value). Interestingly, even in the no EIPH group some patients had a slope of mPAP-LVCO relationship $>3 \mathrm{~mm} \mathrm{Hg} / \mathrm{L} / \mathrm{min}(\mathrm{n}=5,21 \%)$. As mentioned in the Limitations of the study section, this could be related to the method of assessment of LVCO that can lead to an underestimation of LVC and thus an overestimation of the slope of mPAP-LVCO relationship. However, our results are in line with recent paper from Gargani et al. [19] reporting a change of $3.1 \pm 2.7 \mathrm{~mm} \mathrm{Hg} / \mathrm{L} / \mathrm{min}$ in the subset of patients with exercise $\mathrm{SPAP}<50 \mathrm{~mm} \mathrm{Hg}$.

Regarding the exercise protocol, an increment of $25 \mathrm{~W}$ may seem to be excessive for chronic ill patients. However, this protocol is in line with other studies on SSc $[19,42]$ and also on other fields of research such as valvular heart diseases [17,18], congenital heart disease [43] or pulmonary arterial hypertension [44]. In addition, a dynamic protocol is necessary to avoid the increase in systemic vascular resistance and abrupt change in intra-thoracic pressure occurring during resistive exercise and that can lead to unpredictable effects on the pulmonary circulation [4].

\section{Conclusion}

EIPH is frequent in patients with SSc (47\%) and may be detected using exercise echocardiography. In patients with EIPH, our results suggest the potential role of increased exercise LV filling pressure and increased PVR. Exercise echocardiography could be useful for the 
screening and the understanding of the pathophysiological mechanisms leading to PH in patients with SSc. Further studies are needed to confirm these results and their impact on the outcome.

\section{Source of funding}

Damien Voilliot is supported by a research grant of the European Association of Cardiovascular Imaging.

\section{Acknowledgments}

We specially thank Carmine Celentano for his precious help and support for investigations in this study.

\section{References}

[1] Gabrielli A, Avvedimento EV, Krieg T. Scleroderma. N Engl J Med 2009;360:1989-2003.

[2] Lefèvre G, Dauchet L, Hachulla E, et al. Survival and prognostic factors in systemic sclerosis-associated pulmonary hypertension: a systematic review and metaanalysis. Arthritis Rheum 2013;65:2412-23.

[3] Schwaiger JP, Khanna D, Gerry Coghlan J. Screening patients with scleroderma for pulmonary arterial hypertension and implications for other at-risk populations. Eur Respir Rev Off J Eur Respir Soc 2013;22:515-25.

[4] Naeije R, Vanderpool R, Dhakal BP, et al. Exercise-induced pulmonary hypertension: physiological basis and methodological concerns. Am J Respir Crit Care Med 2013:187:576-83.

[5] Lewis GD, Bossone E, Naeije R, et al. Pulmonary vascular hemodynamic response to exercise in cardiopulmonary diseases. Circulation 2013;128:1470-9.

[6] Saggar R, Sitbon O. Hemodynamics in pulmonary arterial hypertension: current and future perspectives. Am J Cardiol 2012;110:9S-15S.

[7] Alkotob ML, Soltani P, Sheatt MA, et al. Reduced exercise capacity and stress-induced pulmonary hypertension in patients with scleroderma. Chest 2006;130:176-81.

[8] Codullo V, Caporali R, Cuomo G, et al. Stress Doppler echocardiography in systemic sclerosis: evidence for a role in the prediction of pulmonary hypertension. Arthritis Rheum 2013;65:2403-11.

[9] Collins N, Bastian B, Quiqueree L, Jones C, Morgan R, Reeves G. Abnormal pulmonary vascular responses in patients registered with a systemic autoimmunity database: Pulmonary Hypertension Assessment and Screening Evaluation using stress echocardiography (PHASE-I). Eur J Echocardiogr J Work Group Echocardiogr Eur Soc Cardiol 2006:7:439-46.

[10] Meune C, Vignaux O, Kahan A, Allanore Y. Heart involvement in systemic sclerosis: evolving concept and diagnostic methodologies. Arch Cardiovasc Dis 2010;103:46-52.

[11] Wells AU, Hansell DM, Rubens MB, et al. Fibrosing alveolitis in systemic sclerosis. Bronchoalveolar lavage findings in relation to computed tomographic appearance. Am J Respir Crit Care Med 1994;150:462-8.

[12] Steen VD, Medsger Jr TA. Severe organ involvement in systemic sclerosis with diffuse scleroderma. Arthritis Rheum 2000:43:2437-44.

[13] Hager WD, Collins I, Tate JP, et al. Exercise during cardiac catheterization distinguishes between pulmonary and left ventricular causes of dyspnea in systemic sclerosis patients. Clin Respir J 2013;7:227-36.

[14] Ciurzyński M, Bienias P, Irzyk K, et al. Exaggerated increase of exercise-induced pulmonary artery pressure in systemic sclerosis patients predominantly results from left ventricular diastolic dysfunction. Clin Res Cardiol Off J Ger Card Soc 2013;102:813-20.

[15] Rudski LG, Lai WW, Afilalo J, et al. Guidelines for the echocardiographic assessment of the right heart in adults: a report from the American Society of Echocardiography endorsed by the European Association of Echocardiography, a registered branch of the European Society of Cardiology, and the Canadian Society of Echocardiography. J Am Soc Echocardiogr Off Publ Am Soc Echocardiogr 2010;23:685-713 [quiz 786-788].

[16] Mukerjee D, St George D, Coleiro B, et al. Prevalence and outcome in systemic sclerosis associated pulmonary arterial hypertension: application of a registry approach. Ann Rheum Dis 2003;62:1088-93.

[17] Lancellotti P, Magne J, Donal E, et al. Determinants and prognostic significance of exercise pulmonary hypertension in asymptomatic severe aortic stenosis. Circulation 2012;126:851-9

[18] Magne J, Lancellotti P, Piérard LA. Exercise pulmonary hypertension in asymptomatic degenerative mitral regurgitation. Circulation 2010;122:33-41.
[19] Gargani L, Pignone A, Agoston G, et al. Clinical and echocardiographic correlations of exercise-induced pulmonary hypertension in systemic sclerosis: a multicenter study. Am Heart J 2013;165:200-7.

[20] Argiento P, Vanderpool RR, Mulè M, et al. Exercise stress echocardiography of the pulmonary circulation: limits of normal and sex differences. Chest 2012;142:1158-65.

[21] Argiento $P$, Chesler N, Mulè $M$, et al. Exercise stress echocardiography for the study of the pulmonary circulation. Eur Respir J 2010;35:1273-8.

[22] Lang RM, Bierig M, Devereux RB, et al. Recommendations for chamber quantification. Eur J Echocardiogr J Work Group Echocardiogr Eur Soc Cardiol 2006;7:79-108.

[23] Steen V, Chou M, Shanmugam V, Mathias M, Kuru T, Morrissey R. Exercise-induced pulmonary arterial hypertension in patients with systemic sclerosis. Chest 2008;134:146-51.

[24] Shim CY, Kim S-A, Choi D, et al. Clinical outcomes of exercise-induced pulmonary hypertension in subjects with preserved left ventricular ejection fraction: implication of an increase in left ventricular filling pressure during exercise. Heart Br Card Soc 2011;97:1417-24.

[25] Miyoshi H, Oishi Y, Mizuguchi Y, et al. Early predictors of alterations in left atrial structure and function related to left ventricular dysfunction in asymptomatic patients with hypertension. J Am Soc Hypertens JASH 2013;7:206-15.

[26] Di Franco M, Paradiso M, Mammarella A, et al. Diastolic function abnormalities in rheumatoid arthritis. Evaluation by echo Doppler transmitral flow and pulmonary venous flow: relation with duration of disease. Ann Rheum Dis 2000;59:227-9.

[27] Hinchcliff M, Desai CS, Varga J, Shah SJ. Prevalence, prognosis, and factors associated with left ventricular diastolic dysfunction in systemic sclerosis. Clin Exp Rheumatol 2012;30:S30-7.

[28] Kahan A, Allanore Y. Primary myocardial involvement in systemic sclerosis. Rheumatol Oxf Engl 2006;45(Suppl. 4):14-7.

[29] Allanore Y, Meune C. Primary myocardial involvement in systemic sclerosis: evidence for a microvascular origin. Clin Exp Rheumatol 2010;28:48-53.

[30] Bulkley BH, Ridolfi RL, Salyer WR, Hutchins GM. Myocardial lesions of progressive systemic sclerosis. A cause of cardiac dysfunction. Circulation 1976;53:483-90.

[31] James TN. De subitaneis mortibus. VIII. Coronary arteries and conduction system in scleroderma heart disease. Circulation 1974;50:844-56.

[32] Gustafsson R, Mannting F, Kazzam E, Waldenström A, Hällgren R. Cold-induced reversible myocardial ischaemia in systemic sclerosis. Lancet 1989;2:475-9.

[33] Steen VD, Follansbee WP, Conte CG, Medsger Jr TA. Thallium perfusion defects predict subsequent cardiac dysfunction in patients with systemic sclerosis. Arthritis Rheum 1996;39:677-81.

[34] Kahan A, Devaux JY, Amor B, et al. Pharmacodynamic effect of dipyridamole on thallium-201 myocardial perfusion in progressive systemic sclerosis with diffuse scleroderma. Ann Rheum Dis 1986;45:718-25.

[35] Vignaux O, Allanore Y, Meune $C$, et al. Evaluation of the effect of nifedipine upon myocardial perfusion and contractility using cardiac magnetic resonance imaging and tissue Doppler echocardiography in systemic sclerosis. Ann Rheum Dis 2005;64:1268-73.

[36] Nesto RW, Kowalchuk G]. The ischemic cascade: temporal sequence of hemodynamic, electrocardiographic and symptomatic expressions of ischemia. Am J Cardiol 1987;59:23C-30C.

[37] Liu J, Zhang Y Cao T-S, et al. Preferential macrovasculopathy in systemic sclerosis detected by regional pulse wave velocity from wave intensity analysis: comparisons of local and regional arterial stiffness parameters in cases and controls. Arthritis Care Res 2011;63:579-87.

[38] Cheng K-S, Tiwari A, Boutin A, et al. Carotid and femoral arterial wall mechanics in scleroderma. Rheumatol Oxf Engl 2003;42:1299-305.

[39] Kovacs G, Berghold A, Scheidl S, Olschewski H. Pulmonary arterial pressure during rest and exercise in healthy subjects: a systematic review. Eur Respir J 2009;34:888-94

[40] Mahjoub H, Levy F, Cassol M, et al. Effects of age on pulmonary artery systolic pressure at rest and during exercise in normal adults. Eur J Echocardiogr J Work Group Echocardiogr Eur Soc Cardiol 2009;10:635-40.

[41] Launay D, Humbert M, Berezne A, et al. Clinical characteristics and survival in systemic sclerosis-related pulmonary hypertension associated with interstitial lung disease. Chest 2011;140:1016-24.

[42] D'Alto M, Ghio S, D'Andrea A, et al. Inappropriate exercise-induced increase in pulmonary artery pressure in patients with systemic sclerosis. Heart 2011;97:112-7.

[43] Van De Bruaene A, De Meester P, Buys R, et al. Right ventricular load and function during exercise in patients with open and closed atrial septal defect type secundum. Eur J Prev Cardiol 2013;20:597-604

[44] Grünig E, Tiede H, Enyimayew EO, et al. Assessment and prognostic relevance of right ventricular contractile reserve in patients with severe pulmonary hypertension. Circulation 2013;128:2005-15. 